\title{
Modeling of differential rotation in rapidly rotating solar-type stars
}

\author{
H. Hotta and T. Yokoyama \\ Department of Earth and Planetary Science, University of Tokyo, 7-3-1 Hongo, Bunkyo-ku, \\ Tokyo 113-0033, Japan \\ hotta.h@eps.s.u-tokyo.ac.jp
}

Received __; accepted _ _ 


\begin{abstract}
We investigate differential rotation in rapidly rotating solar-type stars by means of an axisymmetric mean field model that was previously applied to the sun. This allows us to calculate the latitudinal entropy gradient with a reasonable physical basis. Our conclusions are as follows: (1) Differential rotation approaches the Taylor-Proudman state when stellar rotation is faster than solar rotation. (2) Entropy gradient generated by the attached subadiabatic layer beneath the convection zone becomes relatively small with a large stellar angular velocity. (3) Turbulent viscosity and turbulent angular momentum transport determine the spatial difference of angular velocity $\Delta \Omega$. (4) The results of our mean field model can explain observations of stellar differential rotation.
\end{abstract}

Subject headings: Sun: interior — Sun: rotation — Stars: interior 


\section{INTRODUCTION}

Our sun has an eleven-year magnetic activity cycle, which is thought to be sustained by the dynamo motion of internal ionized plasma, i.e., a transformation of kinetic energy

to magnetic energy (Parker 1955). Our understanding of the solar dynamo has significantly improved during the past fifty years, and some kinematic studies can now reproduce solar magnetic features such as equatorward migration of sunspots and poleward migration of the magnetic field (Choudhuri et al. 1995; Dikpati \& Charbonneau 1999; Küker et al. 2001; Charbonneau 2005; Hotta \& Yokoyama 2010al,b). The most important mechanism of the solar dynamo is the $\Omega$ effect, the bending of pre-existing poloidal magnetic fields by differential rotation and the generation of toroidal magnetic fields. Thus, the distribution of the differential rotation in the convection zone is a significant factor for the solar dynamo. Using helioseismology, it has recently been shown that the solar internal differential rotation is in a non-Taylor-Proudman state (see review by Thompson et al. 2003), meaning the iso-rotation surfaces are not parallel to the axis.

Based on solar observations, it is known that Ca H-K fluxes can be a signature of stellar chromospheric activity, and such chromospheric signatures are in correlation with magnetic activity. Wilson (1968, 1978) and Baliunas et al. (1995) discuss a class of stars that shows a periodic variation in $\mathrm{Ca} \mathrm{H}-\mathrm{K}$ fluxes, which suggests that they have a magnetic cycle similar to our sun. It is natural to conjecture that such magnetic activity is maintained by dynamo action. Various studies have been conducted to investigate the relationship between stellar angular velocity $\Omega_{0}$ and its latitudinal difference $\Delta \Omega$ i.e., $\Delta \Omega \propto \Omega_{0}^{n}$, where the suggested range of $n$ is $0<n<1$ (Donahue et al. 1996; Reiners \& Schmitt 2003; Barnes et al. 2005). This means that the angular velocity difference $\Delta \Omega$ increases and the relative difference $\Delta \Omega / \Omega_{0}$ decreases with increases in the stellar rotation rate $\Omega_{0}$.

In this paper, we investigate differential rotation in rapidly rotating stars using a mean 
field framework. Our study is based on the work of Rempel (2005b), in which he suggests the importance of the role of the subadiabatic layer below the convection zone in order to maintain a non-Taylor-Proudman state in the Sun. The aim of this paper is to use a mean field model to analyze firstly the dependence of the morphology of differential rotation on stellar angular velocity, and secondly the physical process which determines the observable angular velocity difference $\Delta \Omega$. According to our knowledge, this is the first work which systematically discusses the application of Rempel's (2005b) solar model to stars.

Other research adopts another approach to the use of mean field models for the analysis of differential rotation in rapidly-rotating stars (Kitchatinov \& Rüdiger 1995; Küker \& Stix 2001). In these studies, the non-Taylor-Proudman state is sustained by anisotropy of turbulent thermal conduction. This anisotropy is generated by the effects of stellar rotation on convective turbulence.

Three-dimensional numerical studies on stellar differential rotation also exist (Brown et al. 2008; Miesch \& Toomre 2009). In these studies, they resolve stellar thermal driven convection and can calculate a self-consistent turbulent angular momentum transport and anisotropy of turbulent thermal conductivity. The subadiabatic layer below the convection zone, however, is not included. The effects of anisotropy of turbulent thermal conductivity and the subadiabatic layer are discussed in this paper.

\section{MODEL}

Using numerical settings similar to those of Rempel's (2005b), we solve the axisymmetric hydrodynamic equations in spherical geometry $(r, \theta)$, where $r$ is the radius, and $\theta$ is the colatitude. The basic assumptions are as follows.

1. A mean field approximation is adopted. All processes on the convective scale 
are parameterized. Thus, the coefficients for turbulent viscosity, turbulent heat conductivity, and turbulent angular momentum transport are explicitly given in the equations.

2. The perturbations of the density and pressure associated with differential rotation are small, i.e., $\rho_{1} \ll \rho_{0}$ and $p_{1} \ll p_{0}$. Here $\rho_{0}$ and $p_{0}$ denote the reference state density and pressure respectively, whereas $\rho_{1}$ and $p_{1}$ are the perturbations. We neglect the second-order terms of these quantities. Note that the perturbation of angular velocity $\left(\Omega_{1}\right)$ and meridional flow $\left(v_{r}, v_{\theta}\right)$ are not small.

3. Since the reference state is assumed to be in an energy flux balance, the entropy equation includes only perturbations.

\subsection{Equations}

We do not use the anelastic approximation here. The equations in an inertial frame can be expressed as

$$
\begin{aligned}
& \frac{\partial \rho_{1}}{\partial t}=-\frac{1}{r^{2}} \frac{\partial}{\partial r}\left(r^{2} v_{r} \rho_{0}\right)-\frac{1}{r \sin \theta} \frac{\partial}{\partial \theta}\left(\sin \theta v_{\theta} \rho_{0}\right), \\
& \frac{\partial v_{r}}{\partial t}=-v_{r} \frac{\partial v_{r}}{\partial r}-\frac{v_{\theta}}{r} \frac{\partial v_{r}}{\partial \theta}+\frac{v_{\theta}^{2}}{r}-\frac{1}{\rho_{0}}\left[\rho_{1} g+\frac{\partial p_{1}}{\partial r}\right]+\left(2 \Omega_{0} \Omega_{1}+\Omega_{1}^{2}\right) r \sin ^{2} \theta+\frac{F_{r}}{\rho_{0}}, \\
& \frac{\partial v_{\theta}}{\partial t}=-v_{r} \frac{\partial v_{\theta}}{\partial r}-\frac{v_{\theta}}{r} \frac{\partial v_{\theta}}{\partial \theta}-\frac{v_{r} v_{\theta}}{r}-\frac{1}{\rho_{0}} \frac{1}{r} \frac{\partial p_{1}}{\partial \theta}+\left(2 \Omega_{0} \Omega_{1}+\Omega_{1}^{2}\right) r \sin \theta \cos \theta+\frac{F_{\theta}}{\rho_{0}} \\
& \frac{\partial \Omega_{1}}{\partial t}=-\frac{v_{r}}{r^{2}} \frac{\partial}{\partial r}\left[r^{2}\left(\Omega_{0}+\Omega_{1}\right)\right]-\frac{v_{\theta}}{r \sin ^{2} \theta} \frac{\partial}{\partial \theta}\left[\sin ^{2} \theta\left(\Omega_{0}+\Omega_{1}\right)\right]+\frac{F_{\phi}}{\rho_{0} r \sin \theta}, \\
& \frac{\partial s_{1}}{\partial t}=-v_{r} \frac{\partial s_{1}}{\partial r}-\frac{v_{\theta}}{r} \frac{\partial s_{1}}{\partial \theta}+v_{r} \frac{\gamma \delta}{H_{p}}+\frac{\gamma-1}{p_{0}} Q+\frac{1}{\rho_{0} T_{0}} \operatorname{div}\left(\kappa_{\mathrm{t}} \rho_{0} T_{0} \operatorname{grad} s_{1}\right),
\end{aligned}
$$

where $\Omega_{0}$ is a constant value that represents the angular velocity of the rigidly rotating radiative zone. We set it as a parameter in Table 1, $\gamma$ is the ratio of specific heats, with the value for an ideal gas being $\gamma=5 / 3 . \kappa_{\mathrm{t}}$ is the coefficient of turbulent thermal conductivity. 
$\delta=\nabla-\nabla_{\text {ad }}$ represents superadiabaticity, where $\nabla=d(\ln T) / d(\ln p)$ (see 2.2 ).$g$ denotes gravitational acceleration. Following from this, the perturbation of pressure $p_{1}$ and pressure scale height $H_{p}$ are expressed as

$$
\begin{aligned}
& p_{1}=p_{0}\left(\gamma \frac{\rho_{1}}{\rho_{0}}+s_{1}\right), \\
& H_{p}=\frac{p_{0}}{\rho_{0} g} .
\end{aligned}
$$

$s_{1}$ is dimensionless entropy normalized by the specific heat capacity at constant volume $c_{\mathrm{v}}$. Turbulent viscous force $\mathbf{F}$ follows from

$$
\begin{gathered}
F_{r}=\frac{1}{r^{2}} \frac{\partial}{\partial r}\left(r^{2} R_{r r}\right)+\frac{1}{r \sin \theta} \frac{\partial}{\partial \theta}\left(\sin \theta R_{\theta r}\right)-\frac{R_{\theta \theta}+R_{\phi \phi}}{r}, \\
F_{\theta}=\frac{1}{r^{2}} \frac{\partial}{\partial r}\left(r^{2} R_{r \theta}\right)+\frac{1}{r \sin \theta} \frac{\partial}{\partial \theta}\left(\sin \theta R_{\theta \theta}\right)+\frac{R_{r \theta}-R_{\phi \phi} \cot \theta}{r}, \\
F_{\phi}=\frac{1}{r^{2}} \frac{\partial}{\partial r}\left(r^{2} R_{r \phi}\right)+\frac{1}{r \sin \theta} \frac{\partial}{\partial \theta}\left(\sin \theta R_{\theta \phi}\right)+\frac{R_{r \phi}+R_{\theta \phi} \cot \theta}{r},
\end{gathered}
$$

with the Reynolds stress tensor

$$
R_{i k}=\rho_{0}\left[\nu_{\mathrm{tv}}\left(E_{i k}-\frac{2}{3} \delta_{i k} \operatorname{div} \mathbf{v}\right)+\nu_{\mathrm{tl}} \Lambda_{i k}\right]
$$

Here $\nu_{\mathrm{tv}}$ is the coefficient of turbulent viscosity and $\nu_{\mathrm{tl}}$ is the coefficient of the $\Lambda$ effect (Kitchatinov \& Rüdiger 1995), a non-diffusive angular momentum transport caused by turbulence. $\nu_{\mathrm{tv}}$ and $\nu_{\mathrm{tl}}$ are expected to have the same value, since both effects are caused by turbulence, i.e., thermal driven convection. We discuss this in more detail in $\oiint 2.3$, $E_{i k}$ 
denotes the deformation tensor, which is given in spherical coordinates by

$$
\begin{aligned}
& E_{r r}=2 \frac{\partial v_{r}}{\partial r} \\
& E_{\theta \theta}=2 \frac{1}{r} \frac{\partial v_{\theta}}{\partial \theta}+2 \frac{v_{r}}{r} \\
& E_{\phi \phi}=\frac{2}{r}\left(v_{r}+v_{\theta} \cot \theta\right), \\
& E_{r \theta}=E_{\theta r}=r \frac{\partial}{\partial r}\left(\frac{v_{\theta}}{r}\right)+\frac{1}{r} \frac{\partial v_{r}}{\partial \theta} \\
& E_{r \phi}=E_{\phi r}=r \sin \theta \frac{\partial \Omega_{1}}{\partial r} \\
& E_{\theta \phi}=E_{\phi \theta}=\sin \theta \frac{\partial \Omega_{1}}{\partial \theta} .
\end{aligned}
$$

An expression for the $\Lambda$ effect $\left(\Lambda_{i k}\right)$ is given later. The amount of energy that is converted by the Reynolds stress from kinematic energy to internal energy is given by

$$
Q=\sum_{i, k} \frac{1}{2} E_{i k} R_{i k}
$$

\subsection{Background Stratification}

We use an adiabatic hydrostatic stratification for the spherically symmetric reference state of $\rho_{0}, p_{0}$ and $T_{0}$. Gravitational acceleration is assumed to have $\sim r^{-2}$ dependence, since the radiative zone $\left(r<0.65 R_{\odot}\right)$ has most of the solar mass. This is expressed as,

$$
\begin{aligned}
& \rho_{0}(r)=\rho_{\mathrm{bc}}\left[1+\frac{\gamma-1}{\gamma} \frac{r_{\mathrm{bc}}}{H_{\mathrm{bc}}}\left(\frac{r_{\mathrm{bc}}}{r}-1\right)\right]^{1 /(\gamma-1)}, \\
& p_{0}(r)=p_{\mathrm{bc}}\left[1+\frac{\gamma-1}{\gamma} \frac{r_{\mathrm{bc}}}{H_{\mathrm{bc}}}\left(\frac{r_{\mathrm{bc}}}{r}-1\right)\right]^{\gamma /(\gamma-1)}, \\
& T_{0}(r)=T_{\mathrm{bc}}\left[1+\frac{\gamma-1}{\gamma} \frac{r_{\mathrm{bc}}}{H_{\mathrm{bc}}}\left(\frac{r_{\mathrm{bc}}}{r}-1\right)\right] \\
& g(r)=g_{\mathrm{bc}}\left(\frac{r}{r_{\mathrm{bc}}}\right)^{-2},
\end{aligned}
$$

where $\rho_{\mathrm{bc}}, p_{\mathrm{bc}}, T_{\mathrm{bc}}, H_{\mathrm{bc}}=p_{\mathrm{bc}} /\left(\rho_{\mathrm{bc}} g_{\mathrm{bc}}\right)$ and $g_{\mathrm{bc}}$ denote the values at the base of the convection zone $r=r_{\mathrm{bc}}$ of density, pressure, temperature, pressure scale height and 
gravitational acceleration, respectively. In this study we use $r_{\mathrm{bc}}=0.71 R_{\odot}$, with $R_{\odot}$ representing the solar radius $\left(R_{\odot}=7 \times 10^{10} \mathrm{~cm}\right)$. We adopt solar values $\rho_{\mathrm{bc}}=0.2 \mathrm{~g} \mathrm{~cm}^{-3}$, $p_{\mathrm{bc}}=6 \times 10^{13}$ dyn $\mathrm{cm}^{-2}, T_{\mathrm{bc}}=m p_{\mathrm{bc}} /\left(k_{\mathrm{B}} \rho_{\mathrm{bc}}\right) \sim 1.82 \times 10^{6} \mathrm{~K}$ and $g_{\mathrm{bc}}=5.2 \times 10^{4} \mathrm{~cm} \mathrm{~s}^{-2}$, where $k_{\mathrm{B}}$ is the Boltzmann constant, and $m$ is the mean particle mass. Fig. 1 shows the profiles of background density, pressure and temperature, and gravitational acceleration.

Although the real sun's stratification is not adiabatic in the convection zone, our reference state is valid, since the absolute value of superadiabaticity is small. In order to include the deviation from adiabatic stratification, we assume superadiabaticity $\delta$ has the following profile:

$$
\delta=\delta_{\mathrm{conv}}+\frac{1}{2}\left(\delta_{\mathrm{os}}-\delta_{\mathrm{conv}}\right)\left[1-\tanh \left(\frac{r-r_{\mathrm{tran}}}{d_{\mathrm{tran}}}\right)\right] .
$$

Here $\delta_{\text {os }}$ and $\delta_{\text {conv }}$ denote the values of superadiabaticity in the overshoot region. $r_{\text {tran }}$ and $d_{\text {tran }}$ denote the position and the steepness of the transition toward the subadiabatically stratified overshoot region, respectively. Superadiabaticity in convection zone is define as

$$
\delta_{\mathrm{conv}}=\delta_{\mathrm{c}} \frac{r-r_{\mathrm{sub}}}{r_{\max }-r_{\mathrm{sub}}}
$$

where $r_{\max }$ denotes the location of the upper boundary. We specify $\delta_{\mathrm{os}}=-1.5 \times 10^{-5}$, $r_{\text {tran }}=0.725 R_{\odot}, r_{\text {sub }}=0.8 R_{\odot}$ and $d_{\text {tran }}=d_{\text {sub }}=0.0125 R_{\odot}$ in our simulations. $\delta_{c}$ is took as a free parameter. The entropy gradient can be expressed as

$$
\frac{d s_{0}}{d r}=-\frac{\gamma \delta}{H_{p}}
$$

The third term of eq. (5), $v_{r} \gamma \delta / H_{p}$, includes the effect of deviations from adiabatic stratification. The term indicates that an upflow (downflow) can make negative (positive) entropy perturbations in the subadiabatically stratified layers $(\delta<0)$. 


\subsection{Diffusivity Profile}

We assume the coefficients of turbulent viscosity and thermal conductivity to be constant within the convection zone, and these smoothly connect with the values of the overshoot region. We assume that the diffusivities only depend on the radial coordinate:

$$
\begin{aligned}
& \nu_{\mathrm{tv}}=\nu_{\mathrm{os}}+\frac{\nu_{0 \mathrm{v}}}{2}\left[1+\tanh \left(\frac{r-r_{\mathrm{tran}}+\Delta}{d_{\kappa \nu}}\right)\right] f_{c}(r), \\
& \nu_{\mathrm{tl}}=\frac{\nu_{01}}{2}\left[1+\tanh \left(\frac{r-r_{\mathrm{tran}}+\Delta}{d_{\kappa \nu}}\right)\right] f_{c}(r), \\
& \kappa_{\mathrm{t}}=\kappa_{\mathrm{os}}+\frac{\kappa_{0}}{2}\left[1+\tanh \left(\frac{r-r_{\mathrm{tran}}+\Delta}{d_{\kappa \nu}}\right)\right] f_{c}(r),
\end{aligned}
$$

with

$$
\begin{aligned}
& f_{c}(r)=\frac{1}{2}\left[1+\tanh \left(\frac{r-r_{\mathrm{bc}}}{d_{\mathrm{bc}}}\right)\right], \\
& \Delta=d_{\kappa \nu} \tanh ^{-1}\left(2 \alpha_{\kappa \nu}-1\right),
\end{aligned}
$$

where $\nu_{0 \mathrm{v}}, \nu_{01}$ and $\kappa_{0}$ are the values of the turbulent diffusivities within the convection zone, and $\nu_{\mathrm{os}}$ and $\kappa_{\mathrm{os}}$ are the values in the overshoot region. We specify $\nu_{01}=\kappa_{01}=3 \times 10^{12} \mathrm{~cm}^{2} \mathrm{~s}^{-1}$, $\nu_{\mathrm{os}}=6 \times 10^{10} \mathrm{~cm}^{2} \mathrm{~s}^{-1}$ and $\kappa_{\mathrm{os}}=6 \times 10^{9} \mathrm{~cm}^{2} \mathrm{~s}^{-1}$, and we treat $\nu_{0 \mathrm{v}}$ as a parameter. $\alpha_{\kappa \nu}$ specifies the values of the turbulent diffusivities at $r=r_{\mathrm{tran}}$, i.e., $\nu_{\mathrm{tv}}=\nu_{\mathrm{os}}+\alpha_{\kappa \nu} \nu_{0 \mathrm{v}}$, $\nu_{\mathrm{tl}}=\alpha_{\kappa \nu} \nu_{01}$ and $\kappa_{\mathrm{t}}=\kappa_{\mathrm{os}}+\alpha_{\kappa \nu} \kappa_{0}$ at $r=r_{\mathrm{tran}} . d_{\mathrm{bc}}$ and $d_{\kappa \nu}$ are the widths of transition. We specify $\alpha_{\kappa \nu}=0.1, d_{\mathrm{bc}}=0.0125 R_{\odot}$ and $d_{\kappa \nu}=0.025 R_{\odot}$. As already mentioned, the coefficients for turbulent viscosity and the $\Lambda$ effect are different in our model from those of Rempel's (2005b). There are two reasons for this. One is that we intend to investigate the influence of both effects on stellar differential rotation separately (see \$4.2). The other reason is that the formation of a tachocline in a reasonable amount of time requires a finite value (though small) for the coefficient of turbulent viscosity even in the radiative zone, in which there is likely to be weak turbulence (Rempel 2005b). Fig. 2 shows the profiles of $\nu_{\mathrm{tv}}, \nu_{\mathrm{tl}}$ and $\kappa_{\mathrm{t}}$. 


\subsection{The $\Lambda$ Effect}

In this study we adopt the non-diffusive part of the Reynolds stress, called the $\Lambda$ effect. The $\Lambda$ effect transports angular momentum and generates differential rotation. The $\Lambda$ effect tensors are expressed as

$$
\begin{aligned}
& \Lambda_{r \phi}=\Lambda_{\phi r}=+L(r, \theta) \cos (\theta+\lambda), \\
& \Lambda_{\theta \phi}=\Lambda_{\phi \theta}=-L(r, \theta) \sin (\theta+\lambda),
\end{aligned}
$$

where $L(r, \theta)$ is the amplitude of the $\Lambda$ effect and $\lambda$ is the inclination of the flux vector with respect to the rotational axis. We use for the amplitude of the $\Lambda$ effect the expressions

$$
\begin{aligned}
& f(r, \theta)=\sin ^{l} \theta \cos \theta \tanh \left(\frac{r_{\max }-r}{d}\right), \\
& L(r, \theta)=\Lambda_{0} \Omega_{0} \frac{f(r, \theta)}{\max |f(r, \theta)|},
\end{aligned}
$$

where $d=0.025 R_{\odot} . \lambda$ and $\Lambda_{0}$ are free-parameters. The value of $l$ needs to be equal to or larger than 2 to ensure regularity near the pole, so we set $l=2$. The $\Lambda$ effect does not depend on $v_{r}, v_{\theta}$ or $\Omega_{1}$, meaning it is a stationary effect. We emphasize that the $\Lambda$ effect depends on stellar angular velocity $\Omega_{0}$, since the $\Lambda$ effect is generated by turbulence and Coriolis force. The more rapidly the star rotates, the more angular momentum the $\Lambda$ effect can transport. The dependence of $\Lambda_{0}$ and $\lambda$ on stellar angular velocity is discussed in $\$ 4.3$.

\subsection{Numerical Settings}

Using the modified Lax-Wendroff scheme with TVD artificial viscosity (Davis 1984), we solve Equations (11)-(5) numerically for the northern hemisphere of the meridional plane in $0.65 R_{\odot}<r<0.93 R_{\odot}$ and $0<\theta<\pi / 2$. We use a uniform resolution of 200 points in the radial direction and 400 points in the latitudinal direction in all of our simulations. Each

simulation run is conducted until it reaches a stationary state. All the variables $\rho_{1}, v_{r}, v_{\theta}$, 
$\Omega_{1}$ and $s_{1}$ are equal to zero in the initial condition. At the top boundary $\left(r=0.93 R_{\odot}\right)$ we adopt stress-free boundary conditions for $v_{r}, v_{\theta}$ and $\Omega_{1}$ and set the derivative of $s_{1}$ to zero:

$$
\begin{aligned}
& \frac{\partial v_{r}}{\partial r}=0, \\
& \frac{\partial}{\partial r}\left(\frac{v_{\theta}}{r}\right)=0, \\
& \frac{\partial \Omega_{1}}{\partial r}=0, \\
& \frac{\partial s_{1}}{\partial r}=0 .
\end{aligned}
$$

The boundary conditions for $v_{r}, v_{\theta}$ and $s_{1}$ at the lower boundary $\left(r=0.65 R_{\odot}\right)$ are the same as those at the top boundary. Differential rotation connects with the rigidly rotating core at the lower boundary, so we adopt $\Omega_{1}=0$ there. At both radial boundaries, we set $\rho_{1}$ to make the right side of eq. (2) equal zero. At the pole and the equator $(\theta=0$ and $\pi / 2)$ we use the symmetric boundary condition:

$$
\begin{aligned}
& \frac{\partial \rho_{1}}{\partial \theta}=0, \\
& \frac{\partial \Omega_{1}}{\partial \theta}=0, \\
& \frac{\partial v_{r}}{\partial \theta}=0, \\
& v_{\theta}=0, \\
& \frac{\partial s_{1}}{\partial \theta}=0 .
\end{aligned}
$$

Due to the low Mach number of the expected flows, a direct compressible simulation is problematic, so adopting the same technique as Rempel (2005b), we reduce the speed of sound by multiplying the right side of eq. (11) by $1 / \zeta^{2}$. The equation of continuity is therefore replaced with

$$
\frac{\partial \rho_{1}}{\partial t}+\frac{1}{\zeta^{2}} \operatorname{div}\left(\rho_{0} \mathbf{v}\right)=0 .
$$

The speed of sound then becomes $\zeta$ times smaller than the original speed. We use $\zeta=200$ in all our calculations. This technique can be used safely in our present study since we 
only discuss stationary states, so the factor $\zeta$ becomes unimportant. The validity of this technique is carefully discussed by Rempel (2005b). We test our code by reproducing the results presented by Rempel (2005b) and check the numerical convergence by runs with different grid spacings. After checking and cleaning up at every time step, conservation of total mass, total angular momentum and total energy are maintained through the simulation runs.

\section{Stellar Differential Rotation and the Taylor-Proudman Theorem}

In this section, based on the work of Rempel (2005b), we explain how the subadiabatically stratified region can generate solar-like differential rotation. The $\phi$ component of the vorticity equation can be expressed as

$$
\frac{\partial \omega_{\phi}}{\partial t}=[\ldots]+r \sin \theta \frac{\partial \Omega^{2}}{\partial z}-\frac{g}{\gamma r} \frac{\partial s_{1}}{\partial \theta}
$$

where $\Omega=\Omega_{0}+\Omega_{1}$, and the $z$ axis represents the rotational axis. The inertial term and the diffusion term are neglected. If the last term of eq. (45) is zero, meaning there is no variation in entropy in the latitudinal direction, then $\partial \Omega^{2} / \partial z=0$ in a stationary state, which is the Taylor-Proudman state. Solar-like differential rotation is generated in four stages.

1. In the northern hemisphere, the $\Lambda$ effect transports angular momentum in the negative $z$ direction and generates a negative $\partial \Omega^{2} / \partial z$.

2. The negative $\partial \Omega^{2} / \partial z$ generates a negative $\omega_{\phi}$ due to Coriolis force. This counterclockwise meridional flow corresponds to a negative $v_{r}$ (downflow) at high latitudes and a positive $v_{r}$ (upflow) at low latitudes.

3. As we mentioned in Section 2.2, downflow (upflow) generates positive (negative) entropy perturbations in the subadiabatically stratified layer beneath the convection 
zone $(\delta<0)$. Meridional flow can generate positive entropy perturbations at high latitudes and negative entropy perturbations at low latitudes. Therefore, $\partial s_{1} / \partial \theta$ becomes negative in the overshoot region.

4. The negative $\partial s_{1} / \partial \theta$ also keeps $\partial \Omega^{2} / \partial z$ negative in a stationary state.

The profile of angular velocity in the convection zone is determined by a balance of angular momentum transport from meridional flow and a reduction in meridional flow from buoyancy force at the subadiabatic layer.

\section{RESULTS AND DISCUSSION}

We run simulations for seventeen cases, with Table 1 showing the parameters for each case.

\subsection{Stellar Differential Rotation}

In this section, we discuss the cases with angular velocities up to 16 times the solar value (represented by $\Omega_{\odot}$ ), placing an emphasis on the morphology of stellar differential rotation. Fig. [3 shows the results of our calculations which correspond to cases 1-5 in Table 1. It is found that the larger stellar angular velocity is, the more likely it is for differential rotation to be in the Taylor-Proudman state, in which the contour lines of the angular velocity are parallel to the rotational axis. To evaluate these results quantitatively, we define a parameter which denotes the morphology of differential rotation. We call it the Non-Taylor-Proudman parameter (hereafter the NTP parameter), which is expressed as

$$
P_{\text {ntp }}=\frac{1}{R_{\odot}^{2} \Omega_{0}^{2}} \int \frac{\partial \Omega_{1}^{2}}{\partial z} d V=\frac{1}{R_{\odot}^{2} \Omega_{0}^{2}} \int\left(\cos \theta \frac{\partial}{\partial r}-\frac{\sin \theta}{r} \frac{\partial}{\partial \theta}\right) \Omega_{1}^{2} d V
$$


where $\Omega_{0}$ is the angular velocity of the radiative zone. When the NTP parameter is zero, differential rotation is in the Taylor-Proudman state. Conversely, differential rotation is far from the Taylor-Proudman state with a large absolute value of the NTP parameter. The value of the NTP parameter with various stellar angular velocities is shown in Fig. 4. The NTP monotonically decreases with increases in stellar angular velocity. These results indicate that with large stellar angular velocity values, differential rotation approaches the Taylor-Proudman state. These results are counter-intuitive, however, since we do not expect differential rotation to approach the Taylor-Proudman state with increasing stellar angular velocity values, since the $\Lambda$ effect, which is a driver of the deviation from the Taylor-Proudman state, is proportional to stellar angular velocity $\Omega_{0}$. These are the most significant findings of this paper, so hereafter in this section we discuss these unexpected results.

We next discuss the temperature difference between the equator and the pole at the base of the convection zone $\left(r=0.71 R_{\odot}\right)$. Since temperature is given as a function of entropy by

$$
T_{1}=\frac{T_{0}}{\gamma}\left[s_{1}+(\gamma-1) \frac{p_{1}}{p_{0}}\right]
$$

and it is easier to measure than entropy, we use it here for discussing the thermal structure of the simulation results in the convection zone. Further, although it is mentioned in $\$ 3$ that entropy gradient is crucial for breaking the Taylor-Proudman constraint, the temperature difference can be used as its proxy. Fig. 5 shows the relationship between stellar angular velocity $\Omega_{0}$ and temperature difference $\Delta T$ at $r=0.71 R_{\odot}$, where $\Delta T=\max \left(T_{1}\left(r_{\mathrm{bc}}, \theta\right)\right)-\min \left(T_{1}\left(r_{\mathrm{bc}}, \theta\right)\right)$. Although the temperature difference monotonously increases with larger stellar angular velocity values, it is not enough to make the rotational profile largely deviate from the Taylor-Proudman state. This can be explained by using the 
thermal wind equation, which is a steady state solution of eq. (45):

$$
0=r \sin \theta \frac{\partial \Omega^{2}}{\partial z}-\frac{g}{\gamma r} \frac{\partial s_{1}}{\partial \theta}
$$

The inertial term and the diffusion term are neglected here. This equation indicates that, for a given value of the NTP, we need an entropy gradient proportional to $\Omega_{0}^{2}$. However, our simulation results show that $\Delta T \propto \Omega_{0}^{0.58}$, which means that as $\Omega_{0}$ increases, the thermal driving force becomes insufficient to push differential rotation away from the Taylor-Proudman state. In other words, the latitudinal entropy gradient in rapidly rotating stars is so small that differential rotation stays close to the Taylor-Proudman state. In our model, meridional flow generates latitudinal entropy gradient at the base of the convection zone. It is conjectured that the insufficient thermal drive is due to a slow meridional flow.

We next investigate the dependence of meridional flow on stellar angular velocity. Fig. 6] shows the radial profile of latitudinal velocity $v_{\theta}$ at $\theta=45^{\circ}$, using the results of cases 1,2 and 9 . In case 2 , stellar angular velocity is twice that of case 1 (the solar value). In case 9, stellar angular velocity is equal to the solar value, and the amplitude of the $\Lambda$ effect is two times the value in case 1. Fig. 6 shows that meridional flow does not depend on stellar angular velocity, while it correlates with the $\Lambda$ effect. Considering eq. (34), the $\Lambda$ effect increases with larger values of stellar angular velocity, since the amplitude of the $\Lambda$ effect is proportional to $\Omega_{0}$. The reason why differential rotation in rapidly rotation stars is close to the Taylor-Proudman state is that meridional flow does not become fast with large stellar angular velocity values.

We interpret the result that the speed of meridional flow does not depend on stellar angular velocity in our model as follows. With large values of stellar angular velocity, more angular momentum is transported by the $\Lambda$-effect (Note that the $\Lambda$-effect is proportional to $\Omega_{0}$ in equation (34) ), so meridional flow obtains more energy from differential rotation. The energy gain does not result in an increase in speed because of the associated enhancement 
of the Coriolis force, which bends the meridional flow in the longitudinal direction. Another explanation is possible in terms of angular momentum transport. The angular momentum fluxes from both meridional flow and the Reynolds stress ( $\Lambda$ effect) must be balanced in a steady state. The former is proportional to $v_{\mathrm{m}} \Omega_{0}$ and the latter is proportional to $\Omega_{0}$, where $v_{\mathrm{m}}$ is the amplitude of meridional flow. Therefore, meridional flow does not depend on stellar angular velocity (Miesch 2005). Our results (Fig. 6) indicate that with a larger stellar angular velocity (case 2), the above mechanism does not generate fast meridional flow. However, this does not occur when only the $\Lambda$ effect is large (case 9).

\subsection{Angular Velocity Difference on the Surface}

In this subsection we discuss angular velocity difference $\Delta \Omega$ at the surface and the relationship between our results and previous observations. We conduct numerical simulations to investigate the physical process which determines $\Delta \Omega$ (cases 1, 6-11). We define angular velocity difference as $\Delta \Omega=\max \left(\Omega_{1}\left(r_{\max }, \theta\right)\right)-\min \left(\Omega_{1}\left(r_{\max }, \theta\right)\right)$.

$\Delta \Omega$ is determined by two opposing effects, a smoothing effect from turbulent viscosity and a steepening effect from the $\Lambda$ effect. In a stationary state these two effects cancel each other out. Latitudinal flux for turbulent viscosity and the $\Lambda$ effect can be written as $\rho_{0} \nu_{0 \mathrm{v}} \Delta \Omega / \Delta \theta$ and $\rho_{0} \nu_{01} \Lambda_{0} \Omega_{0}$, respectively. Because these two have approximately the same value, $\Delta \Omega$ can be estimated as

$$
\Delta \Omega \sim \frac{\nu_{01}}{\nu_{0 \mathrm{v}}} \Lambda_{0} \Omega_{0} \Delta \theta
$$

where $\Delta \theta$ denotes the differential rotation region.

In order to confirm eq. (49), we conduct two sets of simulations, firstly varying the value of turbulent viscosity $\left(\nu_{0 \mathrm{v}}\right)$, and secondly the amplitude of the $\Lambda$ effect $\left(\Lambda_{0}\right)$. Note that the setting for turbulent viscosity does not reflect a real situation, since the coefficients 
of turbulent viscosity and the $\Lambda$ effect should have a common value. Nonetheless, this is necessary for the purpose of our investigation. The simulation results are shown in Figures 7 and 8 . We obtain $\Delta \Omega \propto \nu_{0 \mathrm{v}}^{-0.88}$ and $\Delta \Omega \propto \Lambda_{0}^{1.1}$, which are consistent with eq. (49).

Fig. 9 shows the results of the dependence of $\Delta \Omega$ on $\Omega_{0}$ (Cases 1-5). Asterisks denote the difference at the surface between the equator and the pole, squares show the difference between the equator and the colatitude $\theta=45^{\circ}$, and triangles are the difference between the equator and the colatitude $\theta=60^{\circ}$. The difference at low latitudes (squares and triangles) monotonically increases with stellar angular velocity. However this is not the case for angular velocity difference between the equator and the pole (asterisk). As we discussed in $\$ 4.1$, when stellar rotation velocity is large, the Taylor-Proudman state is achieved, meaning the gradient of angular velocity at the surface concentrates in lower latitudes. Due to this concentration, $\Delta \theta$ becomes smaller in Eq. (49) with larger values of $\Omega_{0}$. Thus, $\Delta \Omega_{0}$ does not show an explicit dependence on $\Omega_{0}$. At low latitudes, $\Delta \theta$ is fixed and the angular velocity difference increases with stellar angular velocity. We obtain $\Delta \Omega \propto \Omega_{0}^{0.43}$ (between the equator and the colatitude $\theta=45^{\circ}$ : squares) and $\Delta \Omega \propto \Omega_{0}^{0.55}$ (between the equator and the colatitude $\theta=60^{\circ}$ : triangles). This indicates that $\Delta \Omega / \Omega_{0}$ decreases with stellar angular velocity. These results are consistent with previous stellar observations (Donahue et al. 1996; Reiners \& Schmitt 2003; Barnes et al. 2005).

\subsection{Variation of $\Lambda$-effect and superadiabaticity}

In this section, we discuss the dependence of meridional flow and differential rotation on free parameters. The parameter set is shown in Table1 (cases 12-17). At first we investigate the influence of the variation of the $\Lambda$ effect. The $\Lambda$ effect has two free parameters, i.e., amplitude $\Lambda_{0}$ and inclination angle $\lambda$ (see \$2.4). Amplitude is thought to become smaller with a larger stellar angular velocity, due to the saturation of the correlations such as $\left\langle v_{r}^{\prime} v_{\phi}^{\prime}\right\rangle$ 
and $\left\langle v_{\theta}^{\prime} v_{\phi}^{\prime}\right\rangle$, where $v_{r}^{\prime}, v_{\theta}^{\prime}$ and $v_{\phi}^{\prime}$ are the radial, latitudinal and longitudinal component turbulent velocities, respectively. Fig. 6] shows that meridional flow becomes slower with a smaller $\Lambda_{0}$, keeping the $\Omega_{0}$ value constant (Case 10). It is clear with the result of $\$ 4.1$ that meridional flow becomes slow with a larger angular velocity when the variation of $\Lambda_{0}$ is included. Brown et al. (2008) reported this effect with their three-dimensional hydrodynamic calculation. When meridional flow is slow, the entropy gradient generated by the subadiabatic layer is small, and differential rotation approaches the Taylor-Proudman state.

The inclination angle is thought to be small with large stellar angular velocity values, since the motion across the rotational axis is restricted (Kichatinov \& Rüdiger 1993). In case 12 , differential rotation with a small inclination angle $\left(\lambda=2.5^{\circ}\right)$ is calculated. Other parameters are the same as case 1 . The radial distribution of meridional flow is shown in Fig. 6. Meridional flow becomes faster with a smaller inclination angle. Because of the efficient angular momentum transport in the $z$ direction when the inclination angle is small, the second term on the right hand side of Eq. (45) is large. This generates a large $\omega_{\phi}$, i.e. fast meridional flow.

In summary, we found that rapid stellar rotation causes two opposing effects on the speed of meridional flow. The speed is reduced by the suppression of $\Lambda_{0}$, while it is enhanced by the angular momentum transport along the axial direction with a smaller $\lambda$. Although the results of the three-dimensional calculation suggest that meridional flow becomes slower with a larger stellar angular velocity, our model cannot draw a conclusion about the speed of meridional flow in rapidly rotating stars.

Next we investigate the influence of superadiabaticity in the convection zone. In cases 13-17, superadiabaticity in the convection zone $\delta_{\mathrm{c}}=1 \times 10^{-6}$. The differences of the NTP parameters with adiabatic and superadiabatic convection zones $\left(P_{\mathrm{ntp}\left(\delta_{\mathrm{c}}=0\right)}-\right.$ 
$\left.P_{\operatorname{ntp}\left(\delta_{\mathrm{c}}=10^{-6}\right)}\right) / P_{\mathrm{ntp}\left(\delta_{\mathrm{c}}=0\right)}$ are shown in Fig. 10. The NTP parameter values with a superadiabatic convection zone are smaller than those with an adiabatic convection zone, since meridional flow in the superadiabatic convection zone makes the entropy gradient small. This result is suggested by Rempel (2005a). Note that the difference between the values of the NTP parameters with an adiabatic and those with a superadiabatic convection zone decreases as the stellar angular velocity increases, since the generation of entropy gradient by the subadiabtic layer becomes ineffective with a larger stellar angular velocity.

\section{SUMMARY}

We have investigated differential rotation in rapidly rotating stars using a mean field model. This work is significant because it can be used as a base for further research on stellar activity cycles, which are most likely caused by the dynamo action of differential rotation in the stellar convection zone.

First, we investigated the morphology of differential rotation in rapidly rotating stars. Although more angular momentum is transported by convection with larger stellar angular velocity, the Coriolis force is stronger than in the solar case, so meridional flow does not be fast. In our model, meridional flow generates latitudinal entropy gradient in the subadiabatically stratified overshoot region. Since the meridional flow is not fast, the entropy gradient is insufficient to move differential rotation far from the Taylor-Proudman state in rapidly rotating stars. As a result, the differential rotation of stars with large stellar angular velocity is close to the Taylor-Proudman state.

The temperature difference between latitudes is probably controlled by two important factors, i.e., the subadiabatic layer below the convection zone and anisotropic heat transport caused by turbulence and rotation. We suggest that the former is important in slow rotators 
like the sun, and the latter in rapid rotators. The subadiabatic-layer effect is included in our model, while anisotropic heat transport is not. We found that the effect of the subadiabatic layer can generate a temperature difference $\Delta T=10 \mathrm{~K}$ in the solar case, which moderately increases with higher rotation speeds, and $\Delta T=30 \mathrm{~K}$ in case $\Omega_{0}=8 \Omega_{\odot}$. The three-dimensional simulations by Brown et al. (2008) include a self-consistent calculation of anisotropy of turbulent thermal transport but not the subadiabatic layer at the bottom boundary. In their calculation $\Delta T$ is most likely smaller than $10 \mathrm{~K}$ in the solar case, since they cannot reproduce the solar differential rotation only with anisotropy of thermal transport. Also, $\Delta T=100 \mathrm{~K}$ in case $\Omega_{0}=5 \Omega_{\odot}$, which is larger than the case with the subadiabatic layer. We speculate that anisotropic heat transport becomes more significant in rapidly rotating stars. There is also a possibility that our calculated entropy gradient at the base of the convection zone can be used as a boundary condition for a self-consistent three dimensional simulation of stellar convection (Miesch et al. 2006). Note that differential rotation in rapidly rotating stars in Küker \& Stix (2001) is not in the Taylor-Proudman state when anisotropy of turbulent thermal conductivity is included. A future study of the simultaneous effects of the attached subadiabatic layer beneath convection zone and anisotoropy of the turbulent thermal conductivity on stellar differential rotation would provide a better understanding of stellar differential rotation.

Next, we investigated angular velocity difference at the surface. The $\Lambda$ effect causes spatial difference in the rotation profile, while turbulent viscosity reduces the difference. Angular velocity difference $\Delta \Omega$ is determined in eq. (49), which is then used to investigate differential rotation in rapidly rotating stars. Since stellar rotation is close to the Taylor-Proudman state, and the radiative core is rotating rigidly, differential rotation is concentrated at low latitudes with large stellar angular velocity. This concentration leads to a small $\Delta \theta$ in eq. (49). Therefore, only at low latitudes our model is consistent with stellar observations. 
Our conclusions are as follows: (1) Differential rotation approaches the TaylorProudman state when stellar rotation is faster than solar rotation. (2) Entropy gradient generated by the attached subadiabatic layer beneath the convection zone becomes relatively small with a large stellar angular velocity. (3) Turbulent viscosity and turbulent angular momentum transport determine the spatial difference of angular velocity $\Delta \Omega$. (4) The results of our mean field model can explain observations of stellar differential rotation.

Our future work will focus on the stellar MHD dynamo. Several investigations have been conducted on the stellar dynamo using a kinematic dynamo framework (Dikpati et al. 2001; Charbonneau \& Saar 2001; Moss \& Sokoloff 2009; Jouve et al. 2010). Since, under such a framework, only the magnetic induction equation is solved using a given velocity field, solving a linear equation, such analysis does not give sufficient information on the strength of the dynamo-generated stellar magnetic field. To obtain the full amplitude of the stellar magnetic field, the feedback to the velocity field is required, i.e., an MHD framework. Adopting a similar approach to Rempel (2006), we can use the results of this paper to investigate the strength of the stellar magnetic field. Recent observations of the strength of the magnetic field generated by stellar differential rotation have been conducted using spectroscopy (e.g. Petit et al. 2008). A comparison of these observations and numerical calculations of the stellar dynamo could give new insight into the stellar magnetic field. Finally, our stellar MHD dynamo study would also contribute to the understanding of recent investigations into stellar magnetic cyclic activity periods (Noves et al. 1984; Saar \& Brandenburg (1999).

We are most grateful to Dr. M. Rempel for helpful advice. Numerical computations were carried out at the General-Purpose PC farm in the Center for Computational Astrophysics (CfCA) of the National Astronomical Observatory of Japan. The page charge for this paper is supported by CfCA. We have greatly benefited from the 
proofreading/editing assistance from the GCOE program. 


\section{REFERENCES}

Baliunas, S. L., et al. 1995, ApJ, 438, 269

Barnes, J. R., Cameron, A. C., Donati, J., James, D. J., Marsden, S. C., \& Petit, P. 2005, MNRAS, 357, L1

Brown, B. P., Browning, M. K., Brun, A. S., Miesch, M. S., \& Toomre, J. 2008, ApJ, 689, 1354

Charbonneau, P. 2005, Living Reviews in Solar Physics, 2, 2

Charbonneau, P., \& Saar, S. H. 2001, in Astronomical Society of the Pacific Conference Series, Vol. 248, Magnetic Fields Across the Hertzsprung-Russell Diagram, ed. G. Mathys, S. K. Solanki, \& D. T. Wickramasinghe, 189

Choudhuri, A. R., Schussler, M., \& Dikpati, M. 1995, A\&A, 303, L29

Davis, S. 1984, Icase Report, 84, 20

Dikpati, M., \& Charbonneau, P. 1999, ApJ, 518, 508

Dikpati, M., Saar, S. H., Brummell, N., \& Charbonneau, P. 2001, in Astronomical Society of the Pacific Conference Series, Vol. 248, Magnetic Fields Across the HertzsprungRussell Diagram, ed. G. Mathys, S. K. Solanki, \& D. T. Wickramasinghe, 235

Donahue, R. A., Saar, S. H., \& Baliunas, S. L. 1996, ApJ, 466, 384

Hotta, H., \& Yokoyama, T. 2010a, ApJ, 709, 1009

-. 2010b, ApJ, 714, L308

Jouve, L., Brown, B. P., \& Brun, A. S. 2010, A\&A, 509, A32 
Kichatinov, L. L., \& Rüdiger, G. 1993, A\&A, 276, 96

Kitchatinov, L. L., \& Rüdiger, G. 1995, A\&A, 299, 446

Küker, M., Rüdiger, G., \& Schultz, M. 2001, A\&A, 374, 301

Küker, M., \& Stix, M. 2001, A\&A, 366, 668

Miesch, M. S. 2005, Living Reviews in Solar Physics, 2, 1

Miesch, M. S., Brun, A. S., \& Toomre, J. 2006, ApJ, 641, 618

Miesch, M. S., \& Toomre, J. 2009, Annual Review of Fluid Mechanics, 41, 317

Moss, D., \& Sokoloff, D. 2009, A\&A, 497, 829

Noyes, R. W., Weiss, N. O., \& Vaughan, A. H. 1984, ApJ, 287, 769

Parker, E. N. 1955, ApJ, 122, 293

Petit, P., et al. 2008, MNRAS, 388, 80

Reiners, A., \& Schmitt, J. H. M. M. 2003, A\&A, 398, 647

Rempel, M. 2005a, ApJ, 631, 1286

-. 2005b, ApJ, 622, 1320

—. 2006, ApJ, 647, 662

Saar, S. H., \& Brandenburg, A. 1999, ApJ, 524, 295

Thompson, M. J., Christensen-Dalsgaard, J., Miesch, M. S., \& Toomre, J. 2003, ARA\&A, 41,599

Wilson, O. C. 1968, ApJ, 153, 221 
—. 1978, ApJ, 226, 379

This manuscript was prepared with the AAS IATEX macros v5.2. 

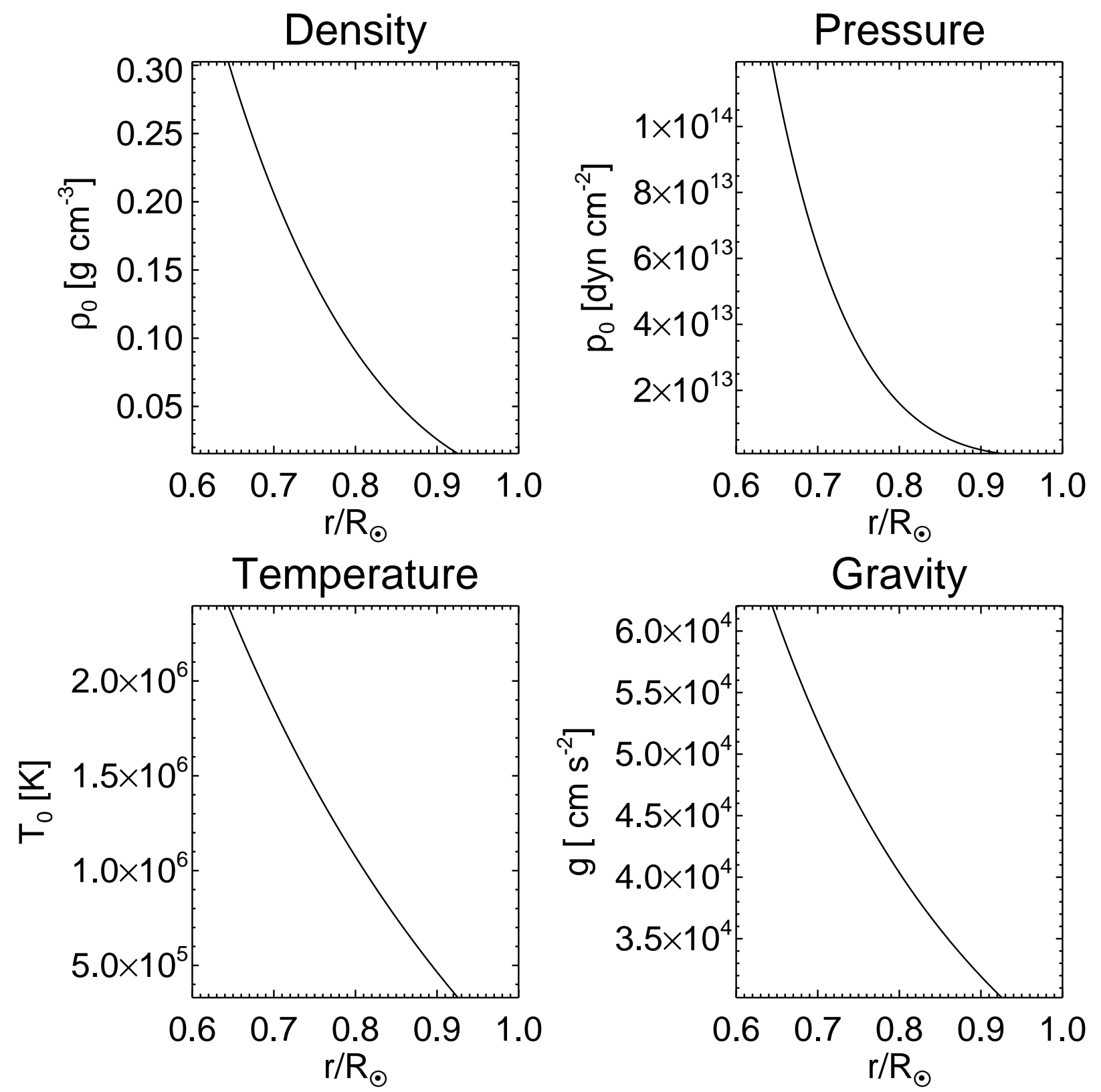

Fig. 1.- Profiles of density, pressure and temperature as a function of radial distance in the reference state. This stratification is adiabatic. 


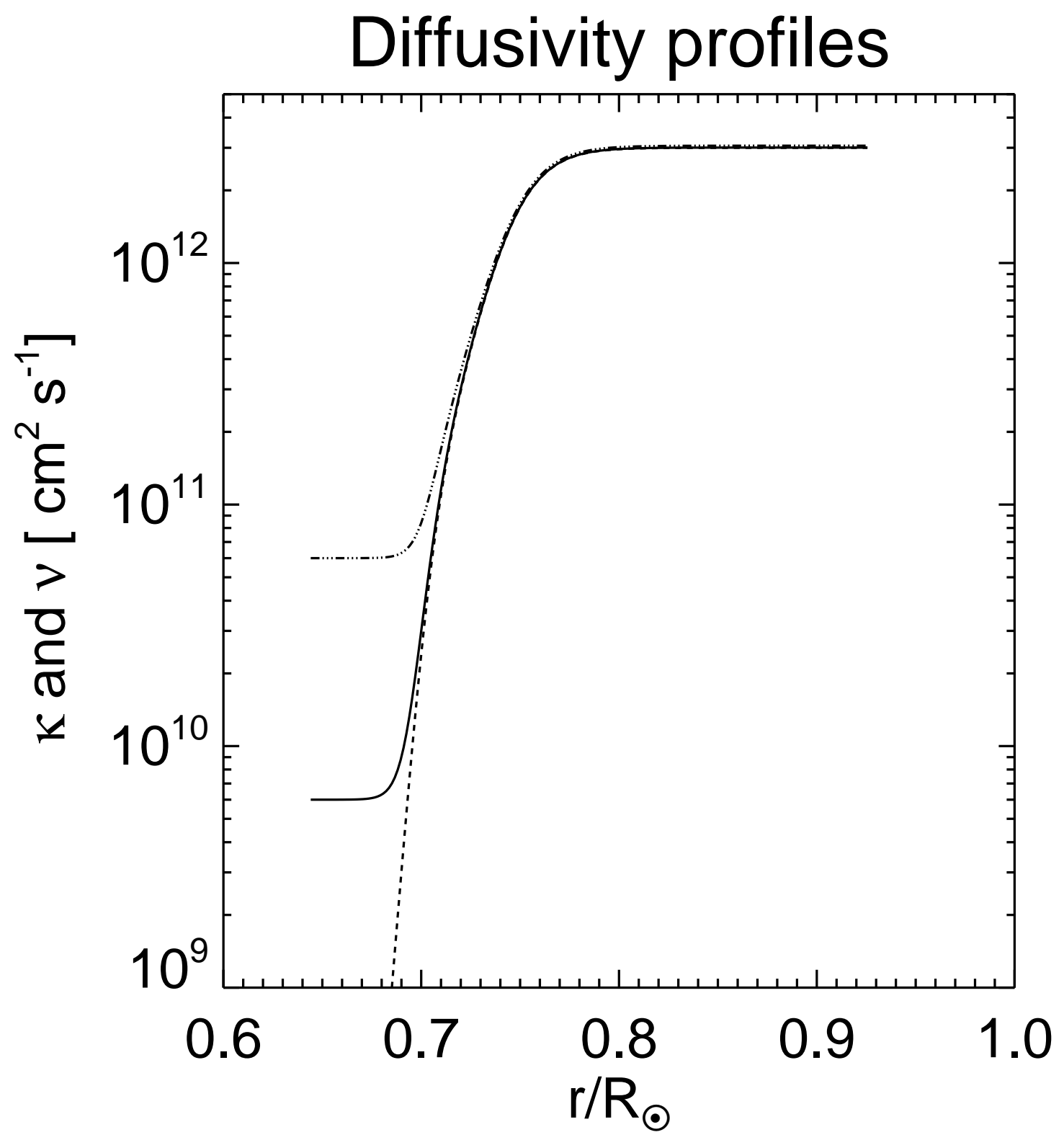

Fig. 2.- Profiles of diffusivity as a function of radial distance for cases 1-5 and 9-11. The solid line denotes the coefficient of turbulent conductivity $\kappa_{\mathrm{t}}$. The dashed line denotes the coefficient of the $\Lambda$ effect $\nu_{\mathrm{tl}}$. The dash and three dots line denotes the coefficient of turbulent viscosity $\nu_{\mathrm{tv}}$. 
Table 1: Significant parameters of the simplified model.

\begin{tabular}{|c|c|c|c|c|c|}
\hline Case & $\Omega_{0}[\mathrm{nHz}]$ & $\nu_{0 \mathrm{v}}\left[\mathrm{cm}^{2} \mathrm{~s}^{-1}\right]$ & $\Lambda_{0}$ & $\lambda$ & $\delta_{\mathrm{c}}$ \\
\hline 1 & $1 \Omega_{\odot}=430$ & $3 \times 10^{12}$ & 1 & $15^{\circ}$ & 0 \\
\hline 2 & $2 \Omega_{\odot}=860$ & $3 \times 10^{12}$ & 1 & $15^{\circ}$ & 0 \\
\hline 3 & $4 \Omega_{\odot}=1720$ & $3 \times 10^{12}$ & 1 & $15^{\circ}$ & 0 \\
\hline 4 & $8 \Omega_{\odot}=3440$ & $3 \times 10^{12}$ & 1 & $15^{\circ}$ & 0 \\
\hline 5 & $16 \Omega_{\odot}=6880$ & $3 \times 10^{12}$ & 1 & $15^{\circ}$ & 0 \\
\hline 6 & $1 \Omega_{\odot}=430$ & $12 \times 10^{12}$ & 1 & $15^{\circ}$ & 0 \\
\hline 7 & $1 \Omega_{\odot}=430$ & $6 \times 10^{12}$ & 1 & $15^{\circ}$ & 0 \\
\hline 8 & $1 \Omega_{\odot}=430$ & $1.5 \times 10^{12}$ & 1 & $15^{\circ}$ & 0 \\
\hline 9 & $1 \Omega_{\odot}=430$ & $3 \times 10^{12}$ & 2 & $15^{\circ}$ & 0 \\
\hline 10 & $1 \Omega_{\odot}=430$ & $3 \times 10^{12}$ & 0.5 & $15^{\circ}$ & 0 \\
\hline 11 & $1 \Omega_{\odot}=430$ & $3 \times 10^{12}$ & 0.25 & $15^{\circ}$ & 0 \\
\hline 12 & $1 \Omega_{\odot}=430$ & $3 \times 10^{12}$ & 1 & $2.5^{\circ}$ & 0 \\
\hline 13 & $1 \Omega_{\odot}=430$ & $3 \times 10^{12}$ & 1 & $15^{\circ}$ & $1 \times 10^{-6}$ \\
\hline 14 & $2 \Omega_{\odot}=860$ & $3 \times 10^{12}$ & 1 & $15^{\circ}$ & $1 \times 10^{-6}$ \\
\hline 15 & $4 \Omega_{\odot}=1720$ & $3 \times 10^{12}$ & 1 & $15^{\circ}$ & $1 \times 10^{-6}$ \\
\hline 16 & $8 \Omega_{\odot}=3440$ & $3 \times 10^{12}$ & 1 & $15^{\circ}$ & $1 \times 10^{-6}$ \\
\hline 17 & $16 \Omega_{\odot}=6880$ & $3 \times 10^{12}$ & 1 & $15^{\circ}$ & $1 \times 10^{-6}$ \\
\hline
\end{tabular}



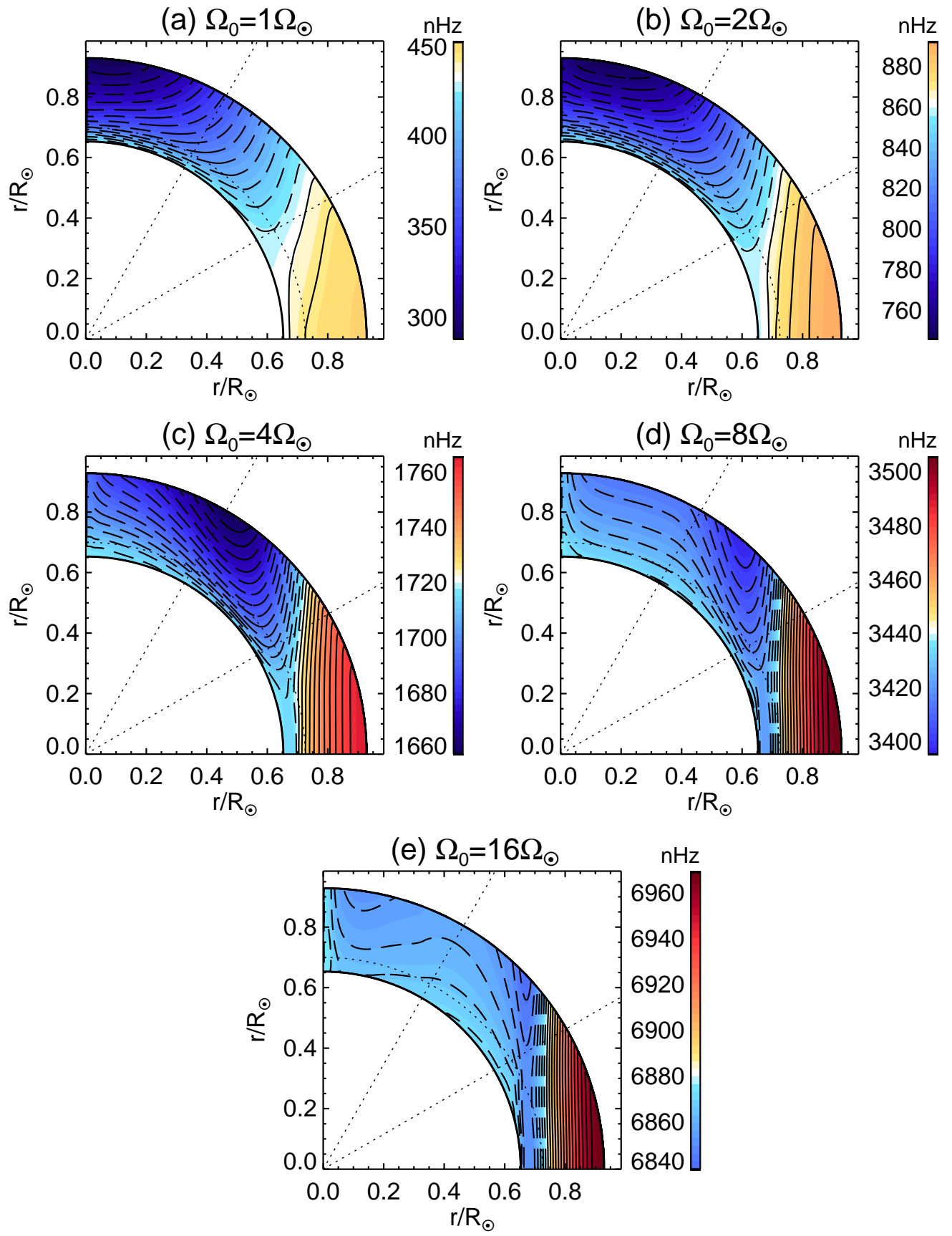

Fig. 3.- Rotation profiles of the simulation results. Panels (a)-(e) correspond to cases 1-5, respectively. The stellar rotation rate for each case is given at the top of each panel. The area of red and solid lines (blue and dashed lines) rotates faster (slower) than the rigidly rotating core at the bottom boundary. Color bars are given for angular velocity $\Omega / 2 \pi=\left(\Omega_{0}+\Omega_{1}\right) / 2 \pi$ in the unit of $\mathrm{nHz}$. The dotted lines in each panel indicate the base of the convection zone $\left(r=0.71 R_{\odot}\right)$ and the colatitudes $\theta=30^{\circ}$ and $\theta=60^{\circ}$. 


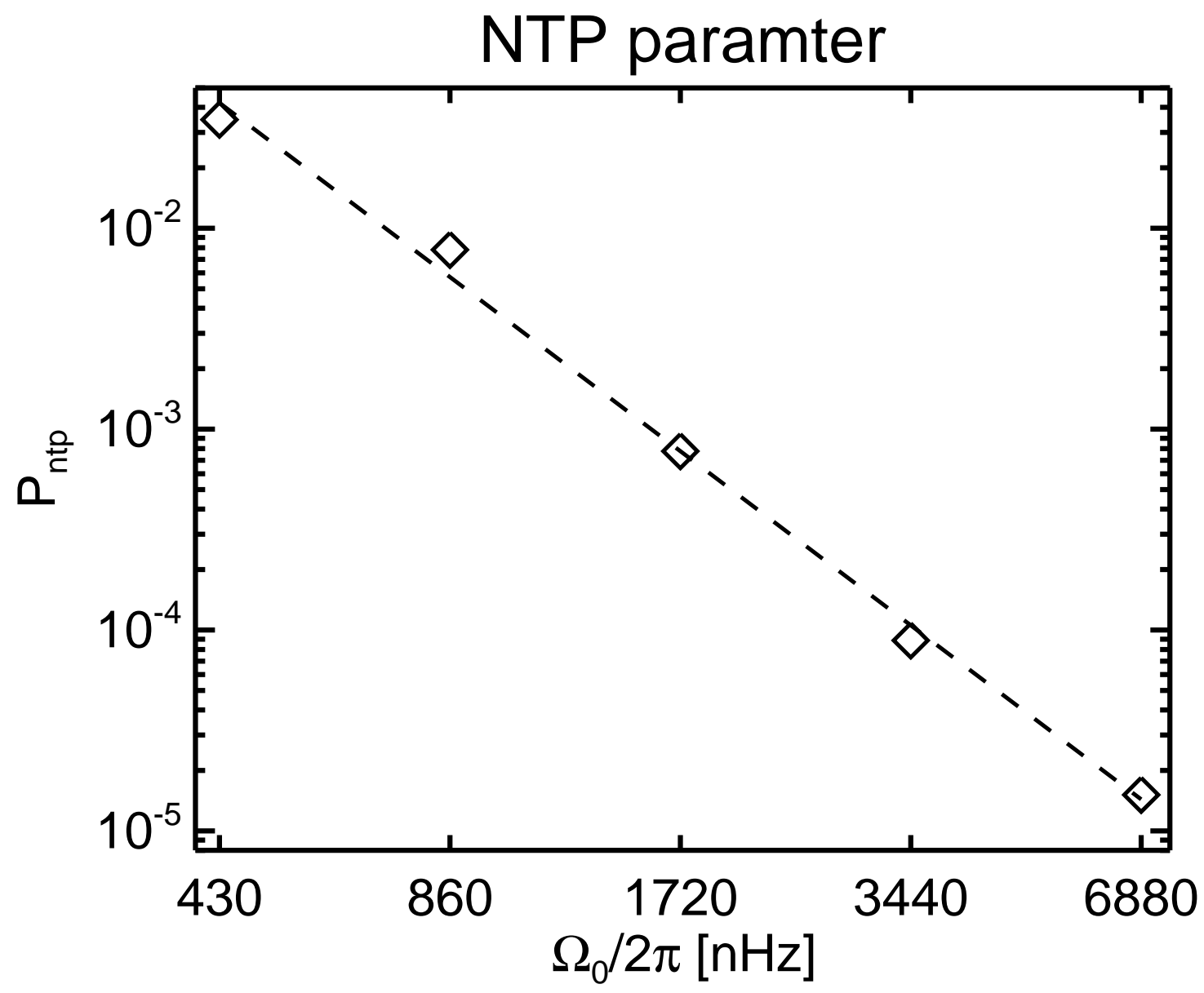

Fig. 4.- NTP parameter as a function of stellar angular velocity $\Omega_{0} / 2 \pi$. The dashed line is the fit to the results showing a power-law function with an index of -2.9 . 


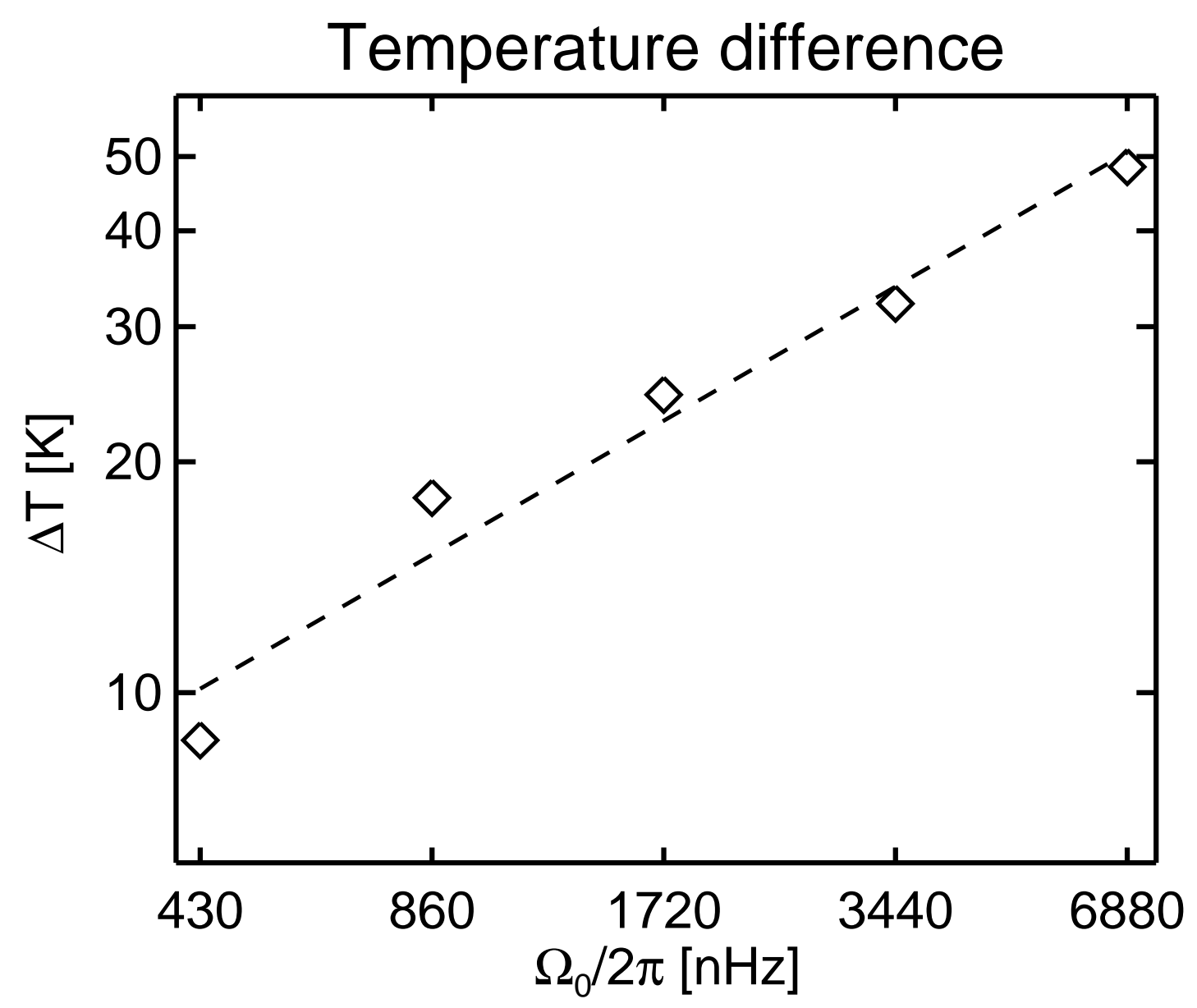

Fig. 5.- Temperature difference at the base of the convection zone $\left(r=0.71 R_{\odot}\right)$ as a function of stellar angular velocity $\left(\Omega_{0} / 2 \pi\right)$. The dashed line is the fit to the results showing a power-law function with an index of 0.58 . 


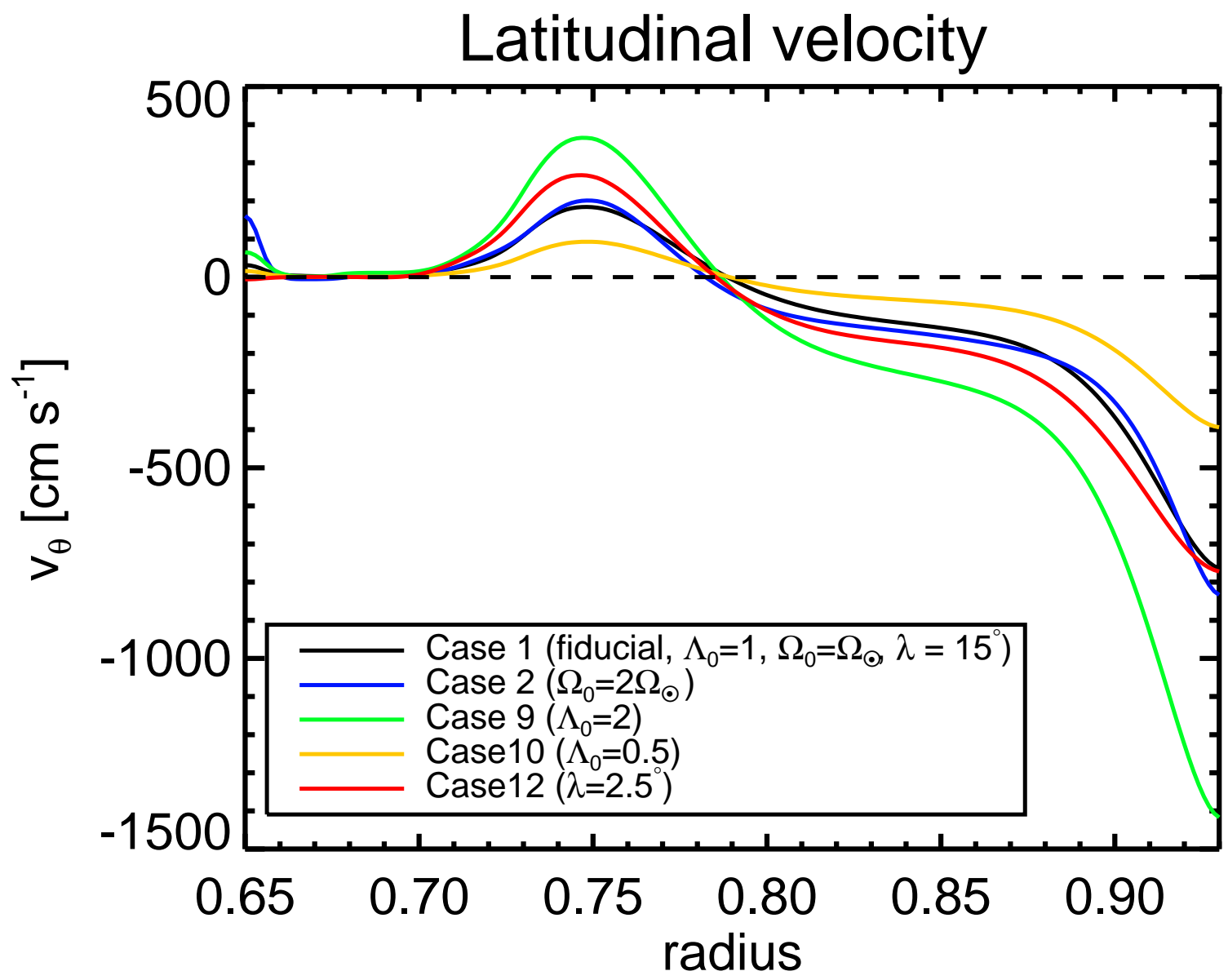

Fig. 6.- Profiles of latitudinal velocity $\left(v_{\theta}\right)$ at colatitude $\theta=45^{\circ}$ as a function of radial distance. In case 1, stellar angular velocity is the solar value, and the amplitude of angular momentum transport $\Lambda_{0}=1$. In case 2 , stellar angular velocity $\Omega_{0}=2 \Omega_{\odot}$. In case 9 and 10 , amplitude of the turbulent angular momentum transport $\Lambda_{0}=2$ and 0.5 , respectively. In case 12 , the inclination angle of $\Lambda$ effect $\lambda=2.5^{\circ}$, and other parameters are the same as case 1 . 


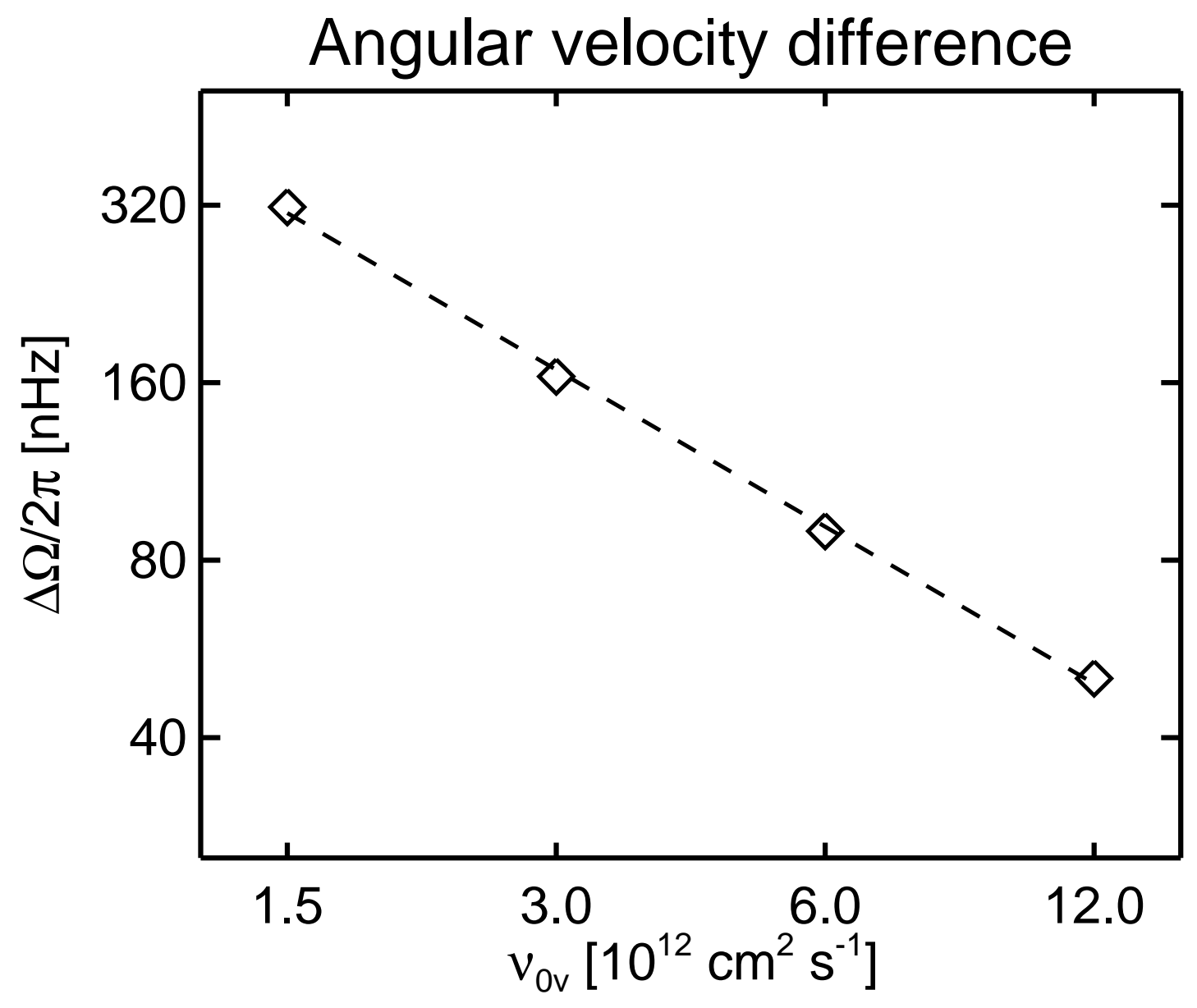

Fig. 7.- Angular velocity difference at the surface as a function of the coefficient of turbulent viscosity $\nu_{0 \mathrm{v}}$. The dashed line is the fit to the results showing a power-law function with an index of -0.88 . 


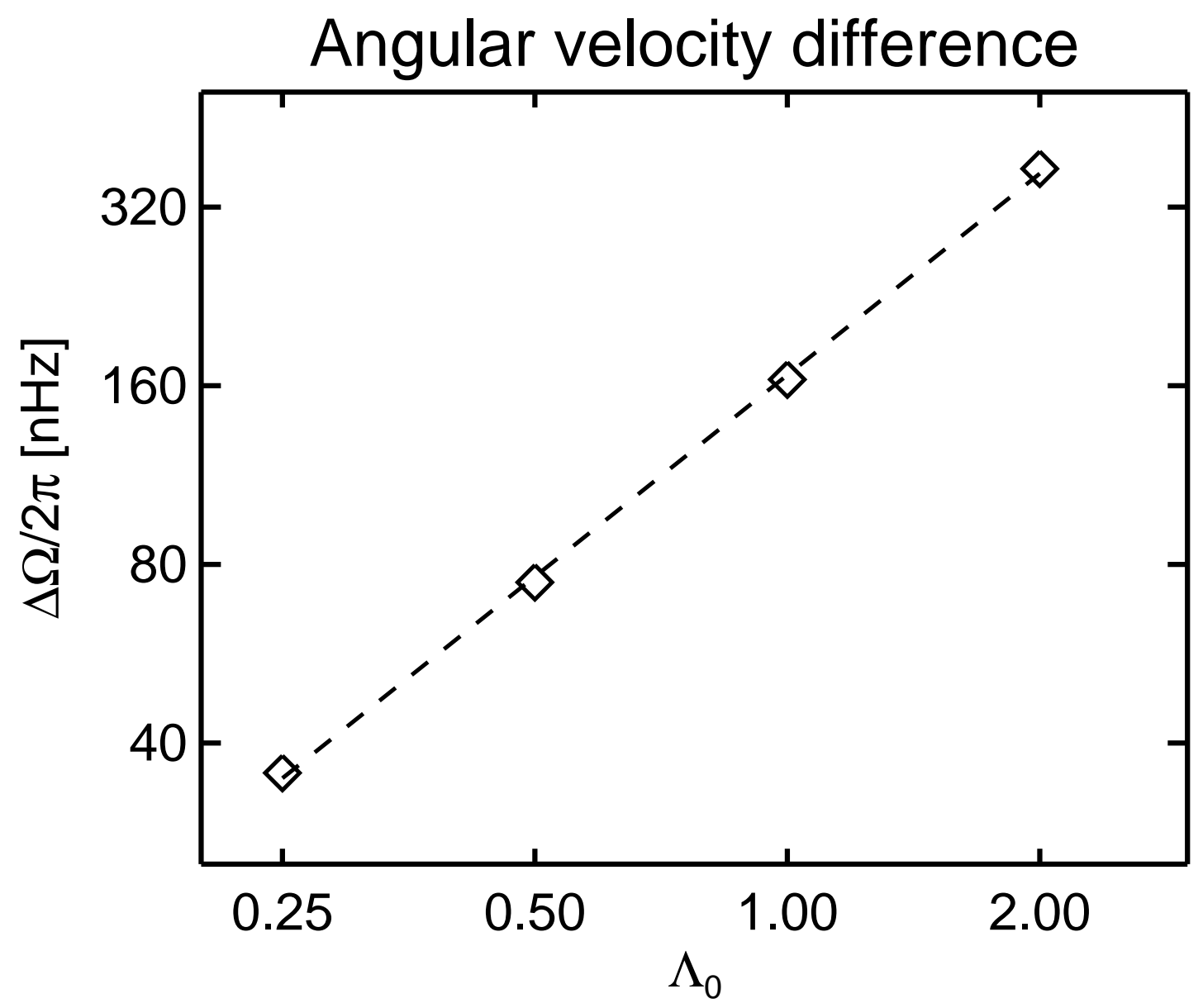

Fig. 8.- Angular velocity difference at the surface as a function of the amplitude of the angular momentum transport $\Lambda_{0}$. The dashed line is the fit to the results showing a powerlaw function with an index of 1.1. 


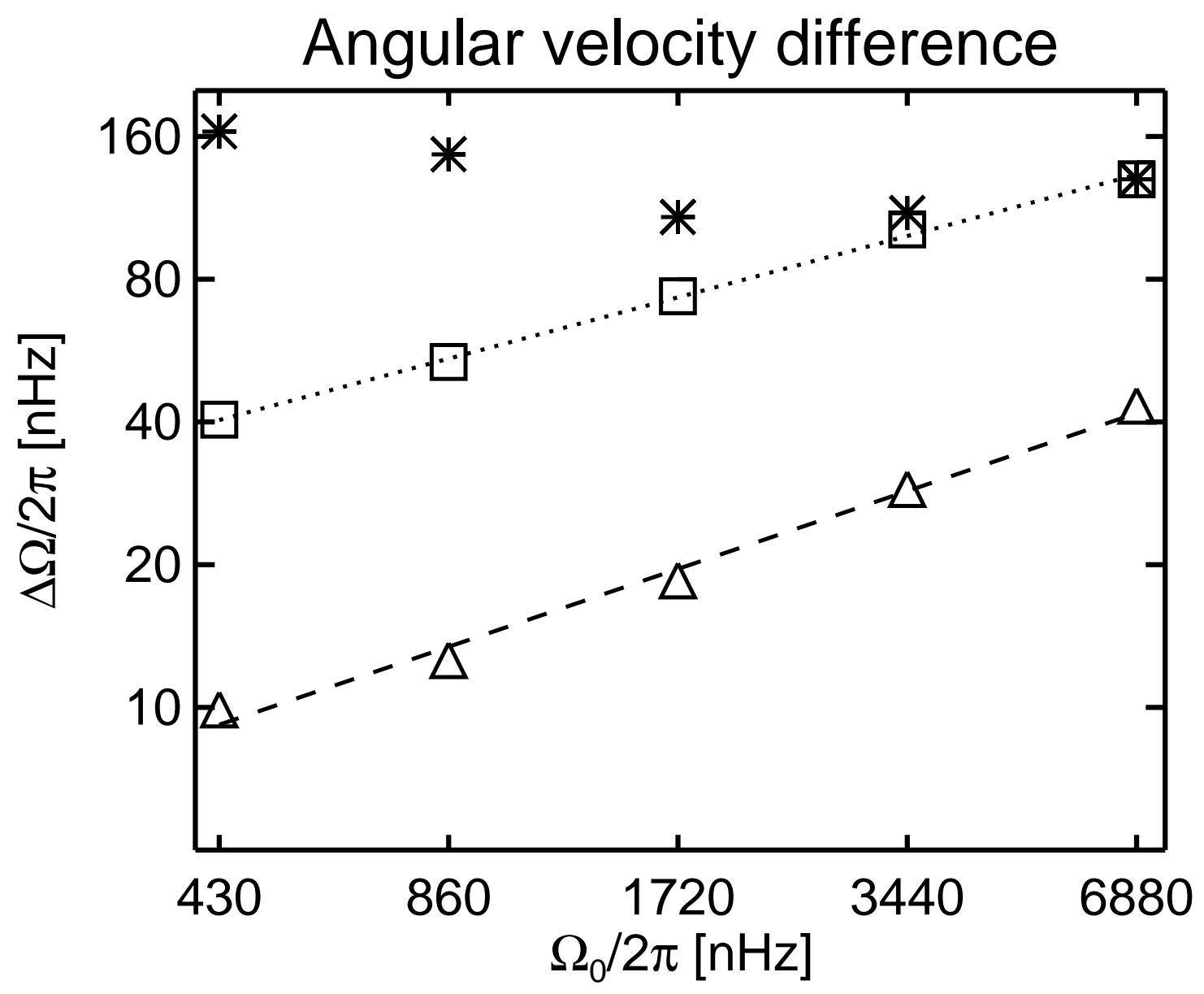

Fig. 9.- Angular velocity difference in three regions. Asterisks, squares and triangles represent the equator and the pole, the equator and the colatitude $\theta=45^{\circ}$ and the equator and the colatitude $\theta=30^{\circ}$, respectively. The dashed and dotted lines are the fits to the results showing a power-law function with indices of 0.43 (squares) and 0.55 (triangles). 


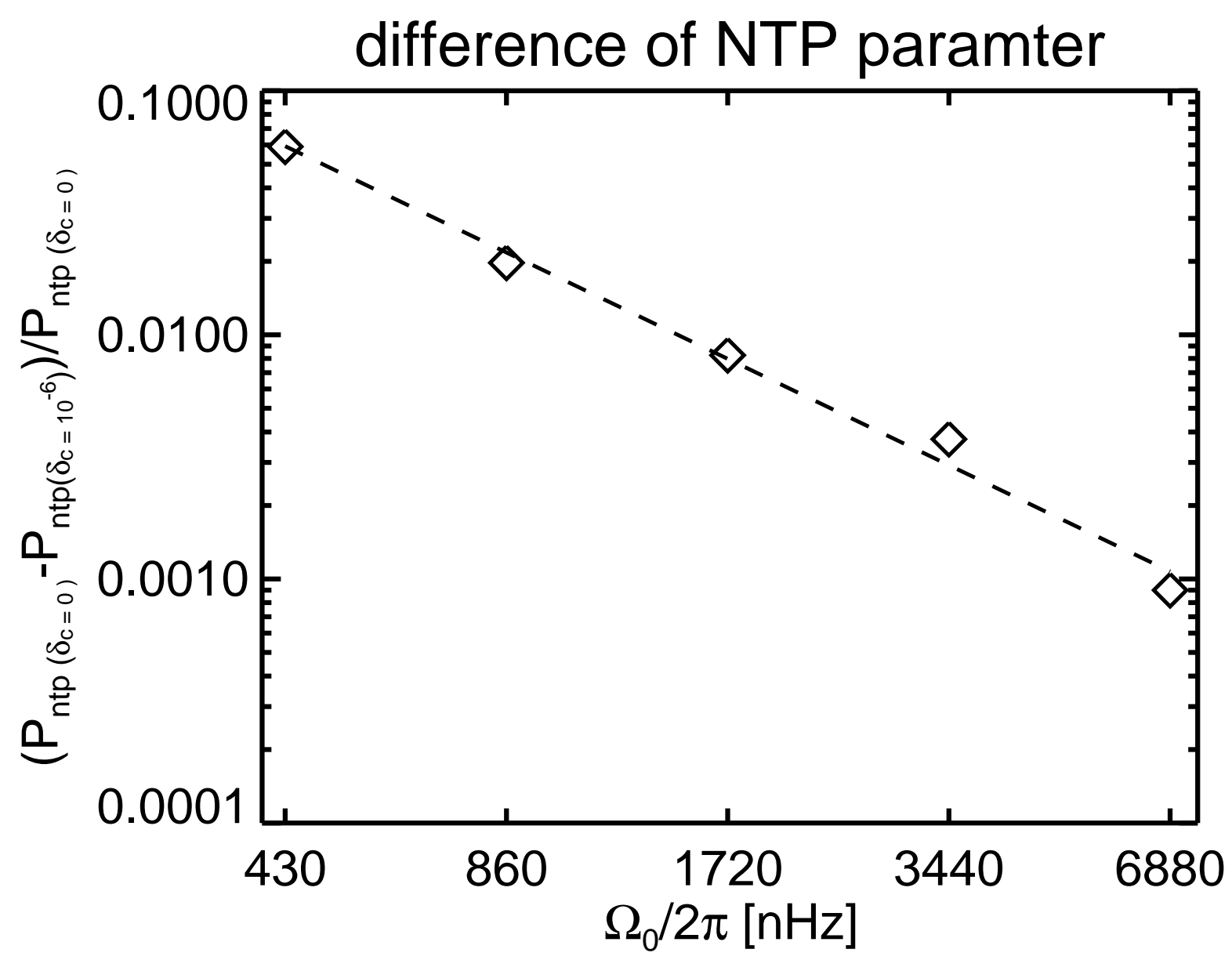

Fig. 10.- The difference of the NTP paramters between cases with adiabatic and superadiabatic convection zone $\left(P_{\operatorname{ntp}\left(\delta_{c}=0\right)}-P_{\operatorname{ntp}\left(\delta_{c}=10^{-6}\right)}\right) / P_{\operatorname{ntp}\left(\delta_{c}=0\right)}$ as a function of stellar angular velocity $\Omega_{0} / 2 \pi$. The dashed line is the fit to the results showing a power-law function with an index of -1.4 . 